\title{
ECMO Support for Influenza A: Retrospective Review of the ELSO Registry Comparing Seasonal and Pandemic Subtypes
}

Erika R O'Neil ( $\square$ erika.oneil.md@gmail.com )

Baylor College of Medicine https://orcid.org/0000-0002-2747-7760

Huiming Lin

Rice University Department of Statistics

Meng Li

Rice University Department of Statistics

Lara Shekerdemian

Baylor College of Medicine

Joseph E. Tonna

University of Utah Health

Ryan P. Barbaro

University of Michigan

Jayvee R. Abella

Medical Informatics Corporation

Peter Rycus

Extracorporeal Life Support Organization

Graeme MacLaren

National University Health System

Marc Anders

Baylor College of Medicine

Peta M.A. Alexander

Boston Childrens Hospital: Boston Children's Hospital

\section{Research}

Keywords: ARDS, extracorporeal membrane oxygenation, respiratory distress

Posted Date: June 28th, 2021

DOl: https://doi.org/10.21203/rs.3.rs-647294/v1 
License: (c) (i) This work is licensed under a Creative Commons Attribution 4.0 International License. Read Full License 


\section{Abstract}

Background: While there is substantial published experience of ECMO during the $\mathrm{H} 1 \mathrm{~N} 1$ pandemic, less is known about the use of ECMO in patients with seasonal influenza A virus. We hypothesized that the severity of illness and survival of patients supported with extracorporeal membrane oxygenation (ECMO) would differ for those with seasonal influenza A vs pandemic H1N1 (H1N1) influenza A.

Methods: Retrospective study of ECMO supported adults (>18 years) with influenza A viral infection reported to the Extracorporeal Life Support Organization (ELSO) Registry between 2009-2019. We describe the incidence and compare characteristics and factors associated with in-hospital survival using a least absolute shrinkage and selection operator regression.

Results: Of 2461 patients supported with ECMO for influenza A, 445 had H1N1 and 2004 had seasonal influenza A. H1N1 was the predominant subtype between 2009-2011. Pandemic H1N1 patients were younger, with more severe illness at ECMO cannulation and higher reported ECMO complications than those with seasonal influenza A. Patient characteristics including younger age and higher weight, and patient management including longer ventilation duration before ECMO were associated with worse survival. ECMO complications were associated with reduced survival. There was no difference in survival to hospital discharge according to influenza subtype after adjusting for other characteristics.

Conclusions: Patients supported with ECMO for pandemic H1N1 were younger, with more severe illness than those supported for seasonal influenza A. Survival to hospital discharge, was associated with patient characteristics, management, and ECMO complications, but was not impacted by the specific influenza A subtype.

\section{Trial registration: N/A}

\section{Background}

In 2009, the H1N1 influenza A pandemic lead to a surge of extracorporeal membrane oxygenation (ECMO) use in critically ill patients with acute respiratory distress syndrome (ARDS) $(1,2,3,4)$. Prior to this, ECMO use in adults with ARDS was relatively rare because two early randomized clinical trials failed to demonstrate a survival benefit $(4,5)$. A large retrospective review of over 1400 adults with ARDS supported on ECMO before 2006 showed $50 \%$ survival (6). The conventional ventilatory support versus extracorporeal membrane oxygenation for severe adult respiratory failure (CESAR) trial in 2009 was the first to demonstrate the safety of ECMO utilization in patients with ARDS (7). More recently, the ECMO to Rescue Lung Injury in Severe ARDS (EOLIA) trial supports a role for ECMO in adult ARDS management (8, 9).

The novel pandemic H1N1 influenza A ( $\mathrm{H} 1 \mathrm{~N} 1)$ virus was associated with increased mortality compared to seasonal influenza A (10-16). Clinical deterioration in young, otherwise well patients during the H1N1 pandemic despite maximal conventional intensive care therapies prompted increased utilization of 
ECMO, with reported survival rates between $35 \%-90 \%(17-28)$. While there is substantial published experience of ECMO during the $\mathrm{H} 1 \mathrm{~N} 1$ pandemic, less is known about the use of ECMO in patients with seasonal influenza A virus.

We hypothesized that the severity of illness and survival of patients supported with ECMO would differ for those with seasonal influenza A vs pandemic H1N1. Against this background, our aims for this project were to 1) describe the incidence of ECMO use over time for H1N1 vs seasonal influenza A; 2) compare characteristics of patients supported on ECMO with H1N1 vs seasonal influenza A; and 3) identify and compare factors associated with survival to hospital discharge in adults with $\mathrm{H} 1 \mathrm{~N} 1 \mathrm{vs}$ seasonal influenza A supported with ECMO.

\section{Methods}

\section{Study Design}

We conducted a multicenter retrospective cohort study using the Extracorporeal Life Support Organization (ELSO) Registry. Adult patients (>18 years) with influenza A during 2009-2019 were eligible for inclusion. Diagnosis of seasonal influenza A and H1N1 were defined by ELSO organism code (Influenza A 63) and/or documentation of International Classification of Diseases (ICD) 9 revision and 10 revision codes (Online Supplement 1).

\section{Variable selection}

Predictor variables were determined a priori, with the inclusion of previously identified factors associated with mortality $(29,30)$. Variables were identified by ICD codes (Online Supplement 1 ) including central nervous system (CNS) dysfunction, immunocompromised state, and shock $(29,30)$. Age, sex, race, weight, pre-ECMO variables including cardiopulmonary arrest or ECPR, duration of mechanical ventilation, renal dysfunction requiring renal replacement therapy, use of neuromuscular blockade agents or inhaled nitric oxide, metabolic buffer infusions, peak inspiratory ventilation pressure (PIP), mean airway pressure (MAP) and partial pressure of arterial carbon dioxide (paCO2) and any known non-pulmonary coinfections were included as covariates. Year of ECMO support, hours of ECMO support, mode of support, and primary indication for support were incorporated as explanatory variables.

Implausible blood gas values were assessed for possible entry in kilopascal instead of millimeters of mercury $(\mathrm{mmHg})$ using an algorithm to calculate the $\mathrm{pH}$ according to the Henderson-Hasselbalch equation. If the calculated $\mathrm{pH}$ corresponded to the $\mathrm{pH}$ of the source, arterial blood gas values were converted to $\mathrm{mmHg}$ by multiplying them by 7.5. Missing $\mathrm{paCO}_{2}$ were replaced by calculated ones if $\mathrm{pH}$ and $\mathrm{HCO} 3$ were entered; missing $\mathrm{pH}$ values were calculated if $\mathrm{paCO} 2$ and $\mathrm{HCO}_{3}$ were available. Variables with more than $15 \%$ missing data were excluded from the analysis.

\section{Outcome}


The primary outcome of interest was survival to discharge from the ECMO center. The secondary outcomes were complications, which were selected by review of the ELSO International Summary Report 2020 , where variables showed a proportional survival of less than $50 \%$ in adult patients with respiratory ECMO (Online Supplement 2) (30).

\section{Statistical Analysis}

Patient and ECMO characteristics were compared between pandemic H1N1 and seasonal influenza A cohorts using univariate analysis. Variables included demographics, comorbidities, pre-ECMO respiratory status, and complications. Categorical and dichotomous variables were expressed as exact numbers with percentages and analyzed with Fisher's exact or Pearson's chi-square. Continuous variables were expressed as median values with 25th - 75th interquartile ranges (IQR) and analyzed with the WilcoxonMann-Whitney test. Univariate unadjusted logistic regression was used to explore the association of patient characteristics against the primary outcome of survival to hospital discharge and reported as odds ratio (OR) with $95 \%$ confidence intervals $(\mathrm{Cl})$.

We describe the incidence and compare characteristics and factors associated with in-hospital survival by both unadjusted logistic regression and multivariate logistic regression with the least absolute shrinkage and selection operator (LASSO) regularization. We employ the LASSO method to achieve dataadaptive variable selection to build a parsimonious, interpretable model (31). Inference in LASSO is notoriously difficult; to this end, we adopt a recently proposed statistical method to mitigate the randomness in the selection of LASSO that supports principled comparison of mortality between influenza subtypes in the presence of LASSO. In particular, Cls and p-values for ORs in the multivariate analysis are derived by exact post-selection inference to ensure valid inference after variable selection by LASSO (32). We preselected the following variables for prediction of mortality: H1N1 and seasonal influenza A, sex, weight (stratified by interquartile ranges with the highest interquartile weight $110-361 \mathrm{~kg}$ as reference variable), age by $18-49$ years, $50-59$ years, and $\geq 60$ years, mechanical ventilation prior ECMO $<48$ hours, 48 hours to 7 days or $\geq 7$ days, CNS dysfunction, diagnosis of shock or immunocompromised state, neuromuscular blockade prior to ECMO, nitric oxide prior to ECMO, metabolic buffer infusions prior to ECMO, other non-respiratory co-infections, cardiac arrest prior to ECMO, and paCO2 $\geq 75 \mathrm{mmHg}$. For an explanatory model of survival, we added to our prediction model, the year of ECMO support (stratified by pandemic years 2009-2011, years after 2011), hours of ECMO support (stratified by interquartile ranges with variable reference $<146.5$ hours), and complications while on ECMO support.

Statistical significance was defined as a $p$-value $<0.05$. Statistical analyses were carried out using $R$ software (version 3.6.1, R foundation for Statistical Computing).

\section{Results}

Inclusion criteria were met for 2528 patients and after exclusions, 2449 underwent univariate analysis, 2335 patients were included in the predictive analysis, and 2311 patients remained in the final 
explanatory model (Fig. 1).

\section{Patients supported on ECMO with H1N1 vs other influenza A subtypes}

Patients with pandemic H1N1 were differentiated from seasonal influenza A and the incidence was determined (Fig. 2). The frequency of reported ECMO support increased during the years 2009-2011 with pandemic H1N1 as the predominant early subtype, but since 2012 other seasonal influenza A subtypes became the leading viral etiology associated with ECMO support. The number of ECMO centers contributing data to the ELSO registry increased from 164 to 463 during the study period (30). ECMO was provided for 445 patients with $\mathrm{H} 1 \mathrm{~N} 1$ and 2004 patients with seasonal influenza A (Table 1). Patients with H1N1 were younger (41.1 vs 48.0 years, $p<0.0001)$ and more commonly white $(79.3 \%$ vs $64.0 \%, p<$ 0.0001 ). Patients with H1N1 were more frequently ventilated with higher PIP ( 36 vs $33 \mathrm{cmH} 20, p<$ 0.0001 ) and MAP (28 vs $24 \mathrm{cmH} 20, p<0.0001)$, with more frequent use of inhaled nitric oxide (19.8 vs $10.6 \%, p<0.001)$ and neuromuscular blockade ( 55.3 vs $48.6 \%, p=0.01)$. Intubation-to-ECMO time in patients with H1N1 was longer (72 vs 35 hours, $p<0.0001$ ) than in patients with seasonal influenza $A$. More patients with seasonal influenza A received renal replacement therapy prior to ECMO (8.9 vs $4.9 \%$, $p$ $=0.005)$. 
Table 1

Patient demographics, characteristics, management, and features of ECMO run.

\begin{tabular}{|c|c|c|c|c|c|}
\hline & $\begin{array}{l}\text { Influenza } \\
\text { A total } \\
(n=2449)\end{array}$ & $\begin{array}{l}\text { Survived } \\
\mathrm{p}^{*}\end{array}$ & $\begin{array}{l}\text { H1N1 } \\
(n= \\
445)\end{array}$ & $\begin{array}{l}\text { Seasonal } \\
\text { influenza } \\
\text { A } \\
(n= \\
2004)\end{array}$ & $\begin{array}{l}\text { Difference among } \\
\text { H1N1 / Seasonal } \\
\text { influenza A p }\end{array}$ \\
\hline & \multicolumn{2}{|l|}{$\begin{array}{l}N(\%) \text { or } \\
\text { Median } \\
{\left[{ }^{[Q R R}\right]^{+}}\end{array}$} & \multicolumn{2}{|c|}{$\begin{array}{l}\mathbf{N}(\%) \text { or Median } \\
{[\text { [QR] }}\end{array}$} & \\
\hline Survival & $\begin{array}{l}1613 \\
(65.9)\end{array}$ & & $\begin{array}{l}289 \\
(64.9)\end{array}$ & $\begin{array}{l}1324 \\
(66.1)\end{array}$ & 0.66 \\
\hline \multirow[t]{2}{*}{ Age in years } & \multirow{2}{*}{$\begin{array}{l}47.2 \\
{[36.7-} \\
56.3]\end{array}$} & $<.0001$ & \multirow{2}{*}{$\begin{array}{l}41.1 \\
{[30.5-} \\
51.9]\end{array}$} & \multirow{2}{*}{$\begin{array}{l}48.0 \\
{[38.5-} \\
57.3]\end{array}$} & \multirow[t]{2}{*}{$<.0001$} \\
\hline & & $\begin{array}{l}\text { OR } 0.97 \\
{[0.97-0.98]}\end{array}$ & & & \\
\hline \multirow[t]{2}{*}{$18-49$} & \multirow{2}{*}{$\begin{array}{l}1443 \\
(58.5)\end{array}$} & $<.0001$ & \multirow{2}{*}{$\begin{array}{l}318 \\
(71.5)\end{array}$} & \multirow{2}{*}{$\begin{array}{l}1125 \\
(55.8)\end{array}$} & \multirow[t]{2}{*}{$<.0001$} \\
\hline & & $\begin{array}{l}\text { OR } 2.56 \\
{[2.03 ; 3.23]}\end{array}$ & & & \\
\hline $50-59$ & $633(25.8)$ & 0.12 & $\begin{array}{l}99 \\
(22.3)\end{array}$ & $\begin{array}{l}534 \\
(26.5)\end{array}$ & 0.06 \\
\hline$>60$ & $383(15.6)$ & reference & $\begin{array}{l}28 \\
(6.3)\end{array}$ & $\begin{array}{l}357 \\
(17.7)\end{array}$ & $<.0001$ \\
\hline \multicolumn{6}{|l|}{$\operatorname{Sex}^{n=2421}$} \\
\hline Male & $\begin{array}{l}1435 \\
(59.3)\end{array}$ & 0.055 & $\begin{array}{l}252 \\
(56.9)\end{array}$ & $\begin{array}{l}1183 \\
(59.8)\end{array}$ & 0.26 \\
\hline Female & $986(40.7)$ & 0.055 & $\begin{array}{l}191 \\
(43.1)\end{array}$ & $\begin{array}{l}795 \\
(40.2)\end{array}$ & 0.26 \\
\hline \multirow[t]{2}{*}{ Weight in $\mathrm{kg}^{\mathrm{n}=2349}$} & \multirow{2}{*}{$\begin{array}{l}90.0[75.0- \\
110.0]\end{array}$} & 0.002 & \multirow{2}{*}{$\begin{array}{l}92.0 \\
{[79.7-} \\
114.0]\end{array}$} & \multirow{2}{*}{$\begin{array}{l}90.0 \\
{[74.6-} \\
109.5]\end{array}$} & \multirow[t]{2}{*}{0.005} \\
\hline & & $\begin{array}{l}\text { OR } 1.00 \\
{[1.00 ; 1.01]}\end{array}$ & & & \\
\hline
\end{tabular}

* If statistically significant association with survival the odds ratio with confidence intervals are listed.

† Categorical variables expressed in numbers with percentages [n (\%)] and continuous variables in medians with interquartile ranges (median, [IQR]).

CNS: central nervous system, ECMO: extracorporeal membrane oxygenation, ECPR: ECMO cardiopulmonary resuscitation, H1N1: pandemic influenza A H1N1 subtype, IQR: interquartile range, kg: kilograms, $\mathrm{NaH} 2 \mathrm{CO} 3$ : sodium bicarbonate, paCO2: partial pressure of arterial carbon dioxide, PIP: positive inspiratory pressure, THAM: tromethamine, VA; venoarterial, VV: venovenous, VVA: venovenoarterial 


\begin{tabular}{|c|c|c|c|c|c|}
\hline & $\begin{array}{l}\text { Influenza } \\
\text { A total } \\
(\mathrm{n}=2449)\end{array}$ & $\begin{array}{l}\text { Survived } \\
\mathrm{p}^{*}\end{array}$ & $\begin{array}{l}\mathrm{H} 1 \mathrm{N1} \\
(\mathrm{n}= \\
445)\end{array}$ & $\begin{array}{l}\text { Seasonal } \\
\text { influenza } \\
\text { A } \\
(n= \\
2004)\end{array}$ & $\begin{array}{l}\text { Difference among } \\
\text { H1N1 / Seasonal } \\
\text { influenza A p }\end{array}$ \\
\hline$<75 \mathrm{~kg}$ & 641 (26.2) & $\begin{array}{l}0.0005 \\
\text { OR } 0.65 \\
{[0.51 ; 0.83]}\end{array}$ & $\begin{array}{l}85 \\
(19.1)\end{array}$ & $\begin{array}{l}556 \\
(27.7)\end{array}$ & 0.0004 \\
\hline 75-90kg & 610 (24.9) & $\begin{array}{l}0.015 \\
\text { OR } 0.74 \\
{[0.57 ; 0.94]}\end{array}$ & $\begin{array}{l}116 \\
(26.1)\end{array}$ & $\begin{array}{l}494 \\
(24.7)\end{array}$ & 0.54 \\
\hline $90-110 \mathrm{~kg}$ & 547 (22.3) & 0.74 & $\begin{array}{l}108 \\
(24.3)\end{array}$ & $\begin{array}{l}439 \\
(21.9)\end{array}$ & 0.29 \\
\hline$>110 \mathrm{~kg}$ & $100(22.5)$ & reference & $\begin{array}{l}108 \\
(24.3)\end{array}$ & $\begin{array}{l}443 \\
(22.1)\end{array}$ & 0.35 \\
\hline Missing & $100(4.1)$ & 0.81 & $\begin{array}{l}28 \\
(6.3)\end{array}$ & 72 (3.6) & 0.02 \\
\hline \multicolumn{6}{|c|}{$\begin{array}{l}\text { Race / Ethnicity } \\
\mathrm{n}=2352\end{array}$} \\
\hline White & $\begin{array}{l}1569 \\
(66.7)\end{array}$ & reference & $\begin{array}{l}336 \\
(79.3)\end{array}$ & $\begin{array}{l}1233 \\
(64.0)\end{array}$ & $<.0001$ \\
\hline Asian & 335 (14.2) & 0.18 & $\begin{array}{l}34 \\
(8.0)\end{array}$ & $\begin{array}{l}301 \\
(15.6)\end{array}$ & $<.0001$ \\
\hline Hispanic & $189(8.0)$ & 0.12 & $\begin{array}{l}20 \\
(4.7)\end{array}$ & $169(8.8)$ & 0.004 \\
\hline Black & $131(5.6)$ & 0.51 & $\begin{array}{l}21 \\
(5.0)\end{array}$ & $110(5.7)$ & 0.64 \\
\hline Multiple & $105(4.4)$ & 0.75 & $\begin{array}{l}13 \\
(3.1)\end{array}$ & $92(4.8)$ & 0.15 \\
\hline \multicolumn{6}{|c|}{ Ventilation $\mathrm{n}=2069$} \\
\hline \multicolumn{6}{|c|}{ * If statistically significant association with survival the odds ratio with confidence intervals are listed } \\
\hline \multicolumn{6}{|c|}{$\begin{array}{l}\text { † Categorical variables expressed in numbers with percentages [n (\%)] and continuous variables in } \\
\text { medians with interquartile ranges (median, [IQR]). }\end{array}$} \\
\hline \multicolumn{6}{|c|}{$\begin{array}{l}\text { CNS: central nervous system, ECMO: extracorporeal membrane oxygenation, ECPR: ECMO } \\
\text { cardiopulmonary resuscitation, H1N1: pandemic influenza A H1N1 subtype, IQR: interquartile range, } \\
\text { kg: kilograms, NaH2CO3: sodium bicarbonate, paCO2: partial pressure of arterial carbon dioxide, PIP: } \\
\text { positive inspiratory pressure, THAM: tromethamine, VA; venoarterial, VV: venovenous, VVA: veno- } \\
\text { venoarterial }\end{array}$} \\
\hline
\end{tabular}




\begin{tabular}{|c|c|c|c|c|c|}
\hline & $\begin{array}{l}\text { Influenza } \\
\text { A total } \\
(n=2449)\end{array}$ & $\begin{array}{l}\text { Survived } \\
\mathrm{p}^{*}\end{array}$ & $\begin{array}{l}\mathrm{H} 1 \mathrm{~N} 1 \\
(n= \\
445)\end{array}$ & $\begin{array}{l}\text { Seasonal } \\
\text { influenza } \\
\text { A } \\
(n= \\
2004)\end{array}$ & $\begin{array}{l}\text { Difference among } \\
\text { H1N1 / Seasonal } \\
\text { influenza A p }\end{array}$ \\
\hline $\mathrm{PIP}(\mathrm{cmH} 20){ }^{n=1627}$ & $\begin{array}{l}33[30- \\
38]\end{array}$ & 0.33 & $\begin{array}{l}36 \\
{[31-} \\
42]\end{array}$ & $\begin{array}{l}33[29- \\
37]\end{array}$ & $<.0001$ \\
\hline $\begin{array}{l}\mathrm{PIP}(\mathrm{cmH} 20)>42 \\
\mathrm{n}=1627\end{array}$ & $211(13.0)$ & 0.21 & $\begin{array}{l}126 \\
(9.8)\end{array}$ & $85(25.8)$ & $<.0001$ \\
\hline $\begin{array}{l}\text { Mean airway pressure } \\
(\mathrm{cmH20})^{n=996}\end{array}$ & $\begin{array}{l}25[20- \\
29]\end{array}$ & 0.81 & $\begin{array}{l}28 \\
{[23-} \\
33]\end{array}$ & $\begin{array}{l}24[20- \\
28]\end{array}$ & $<.0001$ \\
\hline \multicolumn{6}{|l|}{ Blood gas } \\
\hline \multirow[t]{2}{*}{$\mathrm{pH}^{\mathrm{n}=2104}$} & \multirow{2}{*}{$\begin{array}{l}7.26 \\
{[7.16-} \\
7.34]\end{array}$} & $<.0001$ & \multirow{2}{*}{$\begin{array}{l}7.27 \\
{[7.18-} \\
7.35]\end{array}$} & \multirow{2}{*}{$\begin{array}{l}7.25 \\
{[7.15-} \\
7.34]\end{array}$} & \multirow[t]{2}{*}{0.005} \\
\hline & & $\begin{array}{l}\text { OR } 1.02 \\
{[1.01 ; 1.03]}\end{array}$ & & & \\
\hline \multirow{2}{*}{$\begin{array}{l}\mathrm{paCO} 2(\mathrm{mmHg}) \\
\mathrm{n}=2112\end{array}$} & \multirow{2}{*}{$\begin{array}{l}57[46- \\
71]\end{array}$} & 0.09 & \multirow{2}{*}{$\begin{array}{l}58 \\
{[47-} \\
73]\end{array}$} & \multirow{2}{*}{$\begin{array}{l}56[46- \\
71]\end{array}$} & \multirow[t]{2}{*}{0.06} \\
\hline & & $\begin{array}{l}\text { OR } 1.00 \\
{[1.00 ; 100]}\end{array}$ & & & \\
\hline \multirow{2}{*}{$\begin{array}{l}\mathrm{paCO} 2(\mathrm{mmHg}) \geq 75 \\
\mathrm{n}=2097\end{array}$} & \multirow[t]{2}{*}{$443(21.1)$} & 0.02 & \multirow{2}{*}{$\begin{array}{l}93 \\
(23.5)\end{array}$} & \multirow{2}{*}{$\begin{array}{l}350 \\
(20.6)\end{array}$} & \multirow[t]{2}{*}{0.22} \\
\hline & & $\begin{array}{l}\text { OR } 0.77 \\
{[0.62 ; 0.96]}\end{array}$ & & & \\
\hline \multirow[t]{2}{*}{ Pre-ECMO arrest } & \multirow[t]{2}{*}{$152(6.2)$} & $<.0001$ & \multirow{2}{*}{$\begin{array}{l}23 \\
(5.2)\end{array}$} & \multirow[t]{2}{*}{$129(6.4)$} & \multirow[t]{2}{*}{0.38} \\
\hline & & $\begin{array}{l}\text { OR } 0.32 \\
{[0.23 ; 0.45]}\end{array}$ & & & \\
\hline \multicolumn{6}{|l|}{ Comorbidities } \\
\hline $\begin{array}{l}\text { Non- respiratory co- } \\
\text { infection }\end{array}$ & 277 (11.3) & 0.32 & $\begin{array}{l}115 \\
(25.8)\end{array}$ & $162(8.1)$ & $<.0001$ \\
\hline
\end{tabular}

* If statistically significant association with survival the odds ratio with confidence intervals are listed.

† Categorical variables expressed in numbers with percentages [n (\%)] and continuous variables in medians with interquartile ranges (median, [IQR]).

CNS: central nervous system, ECMO: extracorporeal membrane oxygenation, ECPR: ECMO cardiopulmonary resuscitation, H1N1: pandemic influenza A H1N1 subtype, IQR: interquartile range, kg: kilograms, $\mathrm{NaH} 2 \mathrm{CO} 3$ : sodium bicarbonate, paCO2: partial pressure of arterial carbon dioxide, PIP: positive inspiratory pressure, THAM: tromethamine, VA; venoarterial, VV: venovenous, VVA: venovenoarterial 


\begin{tabular}{|c|c|c|c|c|c|}
\hline & $\begin{array}{l}\text { Influenza } \\
\text { A total } \\
(n=2449)\end{array}$ & $\begin{array}{l}\text { Survived } \\
\mathrm{p}^{*}\end{array}$ & $\begin{array}{l}\mathrm{H} 1 \mathrm{~N} 1 \\
(\mathrm{n}= \\
445)\end{array}$ & $\begin{array}{l}\text { Seasonal } \\
\text { influenza } \\
\text { A } \\
(n= \\
2004)\end{array}$ & $\begin{array}{l}\text { Difference among } \\
\text { H1N1 / Seasonal } \\
\text { influenza A p }\end{array}$ \\
\hline CNS dysfunction & $181(7.4)$ & $\begin{array}{l}<.0001 \\
\text { OR 0.38 } \\
{[0.28 ; 0.52]}\end{array}$ & $\begin{array}{l}45 \\
(10.1)\end{array}$ & $136(6.8)$ & 0.02 \\
\hline $\begin{array}{l}\text { Immunocompromised } \\
\text { state }\end{array}$ & $138(5.6)$ & $\begin{array}{l}<.0001 \\
\text { OR } 0.42 \\
{[0.30 ; 0.60]}\end{array}$ & $\begin{array}{l}19 \\
(4.3)\end{array}$ & $119(5.9)$ & 0.21 \\
\hline Shock & $399(16.2)$ & $\begin{array}{l}<.0001 \\
\text { OR 0.51 } \\
{[0.41 ; 0.63]}\end{array}$ & $\begin{array}{l}54 \\
(12.1)\end{array}$ & $\begin{array}{l}345 \\
(17.2)\end{array}$ & 0.009 \\
\hline Pre-ECMO support & & & & & \\
\hline $\begin{array}{l}\text { Neuro-muscular } \\
\text { blockade }\end{array}$ & $\begin{array}{l}1216 \\
(49.7)\end{array}$ & 0.39 & $\begin{array}{l}246 \\
(55.3)\end{array}$ & $\begin{array}{l}970 \\
(48.4)\end{array}$ & 0.009 \\
\hline Nitric oxide & $300(12.2)$ & 1.00 & $\begin{array}{l}88 \\
(19.8)\end{array}$ & $\begin{array}{l}212 \\
(10.6)\end{array}$ & $<.0001$ \\
\hline $\begin{array}{l}\text { Renal replacement } \\
\text { therapy }\end{array}$ & $200(8.2)$ & 0.07 & $\begin{array}{l}22 \\
(4.9)\end{array}$ & $178(8.9)$ & .005 \\
\hline $\begin{array}{l}\text { Metabolic buffers } \\
\text { (THAM or NaH2CO3) }\end{array}$ & $215(8.8)$ & $\begin{array}{l}<.0001 \\
\text { OR 0.45 } \\
{[0.34 ; 0.60]}\end{array}$ & $\begin{array}{l}41 \\
(9.2)\end{array}$ & $174(8.7)$ & 0.71 \\
\hline $\begin{array}{l}\text { Intubation to time on } \\
\mathrm{ECMO}(\mathrm{hrs})^{\mathrm{n}=2212}\end{array}$ & $\begin{array}{l}39[14- \\
107]\end{array}$ & $\begin{array}{l}0.01 \\
\text { OR 0.998 } \\
{[0.998 ; 0.999]}\end{array}$ & $\begin{array}{l}72 \\
{[24-} \\
152]\end{array}$ & $\begin{array}{l}35[12- \\
97]\end{array}$ & $<.0001$ \\
\hline$<48$ hours & $\begin{array}{l}1212 \\
(49.5)\end{array}$ & reference & $\begin{array}{l}162 \\
(39.2)\end{array}$ & $\begin{array}{l}1050 \\
(58.4)\end{array}$ & $<.0001$ \\
\hline
\end{tabular}

* If statistically significant association with survival the odds ratio with confidence intervals are listed.

† Categorical variables expressed in numbers with percentages [n (\%)] and continuous variables in medians with interquartile ranges (median, [IQR]).

CNS: central nervous system, ECMO: extracorporeal membrane oxygenation, ECPR: ECMO cardiopulmonary resuscitation, H1N1: pandemic influenza A H1N1 subtype, IQR: interquartile range, kg: kilograms, $\mathrm{NaH} 2 \mathrm{CO} 3$ : sodium bicarbonate, paCO2: partial pressure of arterial carbon dioxide, PIP: positive inspiratory pressure, THAM: tromethamine, VA; venoarterial, VV: venovenous, VVA: venovenoarterial 


\begin{tabular}{|c|c|c|c|c|c|}
\hline & $\begin{array}{l}\text { Influenza } \\
\text { A total } \\
(n=2449)\end{array}$ & $\begin{array}{l}\text { Survived } \\
\mathrm{p}^{*}\end{array}$ & $\begin{array}{l}\text { H1N1 } \\
(n= \\
445)\end{array}$ & $\begin{array}{l}\text { Seasonal } \\
\text { influenza } \\
\text { A } \\
(n= \\
2004)\end{array}$ & $\begin{array}{l}\text { Difference among } \\
\text { H1N1 / Seasonal } \\
\text { influenza A p }\end{array}$ \\
\hline $\begin{array}{l}\geq 48 \text { hours and }<7 \\
\text { days }\end{array}$ & $721(29.4)$ & $\begin{array}{l}0.002 \\
\text { OR } 0.74 \\
{[0.61 ; 0.90]}\end{array}$ & $\begin{array}{l}167 \\
(40.4)\end{array}$ & $\begin{array}{l}554 \\
(30.8)\end{array}$ & 0.0002 \\
\hline$\geq 7$ days & 279 (11.4) & $\begin{array}{l}0.001 \\
\text { OR } 0.63 \\
{[0.48 ; 0.83]}\end{array}$ & $\begin{array}{l}84 \\
(20.3)\end{array}$ & $\begin{array}{l}195 \\
(10.8)\end{array}$ & $<.0001$ \\
\hline Missing & 237 (9.7) & 0.11 & $\begin{array}{l}32 \\
(7.2)\end{array}$ & $\begin{array}{l}205 \\
(10.2)\end{array}$ & 0.051 \\
\hline \multicolumn{6}{|l|}{ ECMO mode } \\
\hline W & $\begin{array}{l}2108 \\
(86.1)\end{array}$ & reference & $\begin{array}{l}377 \\
(84.7)\end{array}$ & $\begin{array}{l}1731 \\
(86.4)\end{array}$ & 0.34 \\
\hline VA or WVA & 207 (8.5) & $\begin{array}{l}<.0001 \\
\text { OR 0.32 } \\
{[0.24 ; 0.43]}\end{array}$ & $\begin{array}{l}31 \\
(7.0)\end{array}$ & $176(8.8)$ & 0.26 \\
\hline Conversion & $113(4.6)$ & $\begin{array}{l}<.0001 \\
\text { OR 0.34 } \\
{[0.23 ; 0.49]}\end{array}$ & $\begin{array}{l}30 \\
(6.7)\end{array}$ & $83(4.1)$ & 0.02 \\
\hline Other / Unknown & $21(0.9)$ & 0.48 & $7(1.6)$ & $14(0.7)$ & 0.08 \\
\hline \multicolumn{6}{|l|}{ Support type } \\
\hline Pulmonary & $\begin{array}{l}2290 \\
(93.5)\end{array}$ & reference & $\begin{array}{l}434 \\
(97.5)\end{array}$ & $\begin{array}{l}1856 \\
(92.6)\end{array}$ & $<.0001$ \\
\hline Cardiac & $128(5.2)$ & $\begin{array}{l}<.0001 \\
\text { OR 0.40 } \\
{[0.28 ; 0.57]}\end{array}$ & $8(1.8)$ & $120(6.0)$ & 0.0004 \\
\hline \multicolumn{6}{|c|}{ * If statistically significant association with survival the odds ratio with confidence intervals are listed } \\
\hline \multicolumn{6}{|c|}{$\begin{array}{l}\text { † Categorical variables expressed in numbers with percentages }[\mathrm{n}(\%)] \text { and continuous variables in } \\
\text { medians with interquartile ranges (median, [IQR]). }\end{array}$} \\
\hline $\begin{array}{l}\text { CNS: central nervous } \\
\text { cardiopulmonary res } \\
\text { kg: kilograms, NaH2 } \\
\text { positive inspiratory p } \\
\text { venoarterial }\end{array}$ & $\begin{array}{l}\text { tem, ECMO: } \\
\text { tation, H1N1 } \\
\text { sodium bic } \\
\text { ure, THAM: }\end{array}$ & $\begin{array}{l}\text { tracorporea } \\
\text { bandemic in } \\
\text { bonate, paC } \\
\text { methamine }\end{array}$ & $\begin{array}{l}\text { mbrane } \\
\text { iza A H1 } \\
\text { oartial pr } \\
\text { venoarte }\end{array}$ & $\begin{array}{l}\text { ygenation, } \\
\text { subtype, IC } \\
\text { sure of arte } \\
\text { l, VV: veno }\end{array}$ & $\begin{array}{l}\text { CPR: ECMO } \\
\text { R: interquartile range, } \\
\text { al carbon dioxide, PIP: } \\
\text { enous, VVA: veno- }\end{array}$ \\
\hline
\end{tabular}




\begin{tabular}{|c|c|c|c|c|c|}
\hline & $\begin{array}{l}\text { Influenza } \\
\text { A total } \\
(n=2449)\end{array}$ & $\begin{array}{l}\text { Survived } \\
\mathrm{p}^{*}\end{array}$ & $\begin{array}{l}\text { H1N1 } \\
(n= \\
445)\end{array}$ & $\begin{array}{l}\text { Seasonal } \\
\text { influenza } \\
\text { A } \\
(n= \\
2004)\end{array}$ & $\begin{array}{l}\text { Difference among } \\
\text { H1N1 / Seasonal } \\
\text { influenza A p }\end{array}$ \\
\hline \multirow[t]{2}{*}{ ECPR } & \multirow[t]{2}{*}{$31(1.3)$} & $<.0001$ & \multirow[t]{2}{*}{$3(0.7)$} & \multirow[t]{2}{*}{$28(1.4)$} & \multirow[t]{2}{*}{0.22} \\
\hline & & $\begin{array}{l}\text { OR } 0.14 \\
{[0.06 ; 0.33]}\end{array}$ & & & \\
\hline ECMO hours ${ }^{n=2425}$ & $\begin{array}{l}257[146- \\
440]\end{array}$ & 0.42 & $\begin{array}{l}264 \\
{[157-} \\
417]\end{array}$ & $\begin{array}{l}255 \\
{[144-} \\
449]\end{array}$ & 0.73 \\
\hline$<146.5$ & 607 (24.8) & reference & $\begin{array}{l}101 \\
(23.1)\end{array}$ & $\begin{array}{l}506 \\
(25.5)\end{array}$ & 0.30 \\
\hline \multirow[t]{2}{*}{$146.5-256.0$} & \multirow[t]{2}{*}{597 (24.3) } & $<.0001$ & \multirow{2}{*}{$\begin{array}{l}105 \\
(24.0)\end{array}$} & \multirow{2}{*}{$\begin{array}{l}492 \\
(24.8)\end{array}$} & \multirow[t]{2}{*}{0.76} \\
\hline & & $\begin{array}{l}\text { OR } 2.87 \\
{[2.24 ; 3.68]}\end{array}$ & & & \\
\hline \multirow[t]{2}{*}{$256.0-442.5$} & \multirow[t]{2}{*}{619 (25.3) } & $<.0001$ & \multirow{2}{*}{$\begin{array}{l}136 \\
(31.1)\end{array}$} & \multirow{2}{*}{$\begin{array}{l}483 \\
(24.3)\end{array}$} & \multirow[t]{2}{*}{0.004} \\
\hline & & $\begin{array}{l}\text { OR 2.29 } \\
{[1.81 ; 2.91]}\end{array}$ & & & \\
\hline \multirow[t]{2}{*}{$>442.5$} & \multirow[t]{2}{*}{$602(24.6)$} & 0.0004 & \multirow{2}{*}{$\begin{array}{l}96 \\
(21.9)\end{array}$} & \multirow{2}{*}{$\begin{array}{l}506 \\
(25.5)\end{array}$} & \multirow[t]{2}{*}{0.13} \\
\hline & & $\begin{array}{l}\text { OR } 0.66 \\
{[0.52 ; 0.83]}\end{array}$ & & & \\
\hline Missing & $24(1.0)$ & 0.68 & $7(1.6)$ & $17(0.9)$ & 0.18 \\
\hline \multicolumn{6}{|l|}{$\begin{array}{l}\text { Any complication } \\
\text { reported }\end{array}$} \\
\hline \multirow[t]{2}{*}{ Cardiovascular } & \multirow[t]{2}{*}{384 (15.7) } & $<.0001$ & \multirow{2}{*}{$\begin{array}{l}108 \\
(24.3)\end{array}$} & \multirow{2}{*}{$\begin{array}{l}276 \\
(13.8)\end{array}$} & \multirow[t]{2}{*}{$<.0001$} \\
\hline & & $\begin{array}{l}\text { OR } 0.36 \\
{[0.29 ; 0.46]}\end{array}$ & & & \\
\hline
\end{tabular}

* If statistically significant association with survival the odds ratio with confidence intervals are listed.

† Categorical variables expressed in numbers with percentages [n (\%)] and continuous variables in medians with interquartile ranges (median, [IQR]).

CNS: central nervous system, ECMO: extracorporeal membrane oxygenation, ECPR: ECMO cardiopulmonary resuscitation, H1N1: pandemic influenza A H1N1 subtype, IQR: interquartile range, kg: kilograms, $\mathrm{NaH} 2 \mathrm{CO} 3$ : sodium bicarbonate, paCO2: partial pressure of arterial carbon dioxide, PIP: positive inspiratory pressure, THAM: tromethamine, VA; venoarterial, VV: venovenous, VVA: venovenoarterial 


\begin{tabular}{|c|c|c|c|c|c|}
\hline & $\begin{array}{l}\text { Influenza } \\
\text { A total } \\
(n=2449)\end{array}$ & $\begin{array}{l}\text { Survived } \\
\text { p* }\end{array}$ & $\begin{array}{l}\mathrm{H} 1 \mathrm{~N} 1 \\
(\mathrm{n}= \\
445)\end{array}$ & $\begin{array}{l}\text { Seasonal } \\
\text { influenza } \\
\text { A } \\
(n= \\
2004)\end{array}$ & $\begin{array}{l}\text { Difference among } \\
\text { H1N1 / Seasonal } \\
\text { influenza A p }\end{array}$ \\
\hline Hemorrhagic & $229(9.3)$ & $\begin{array}{l}<.0001 \\
\text { OR } 0.51 \\
{[0.38 ; 0.66]}\end{array}$ & $\begin{array}{l}50 \\
(11.2)\end{array}$ & 179 (8.9) & 0.15 \\
\hline Infectious & $42(1.7)$ & $\begin{array}{l}0.001 \\
\text { OR } 0.23 \\
{[0.12 ; 0.44]}\end{array}$ & $\begin{array}{l}12 \\
(2.7)\end{array}$ & $30(1.5)$ & 0.10 \\
\hline Mechanical & $76(3.1)$ & $\begin{array}{l}0.0008 \\
\text { OR } 0.45 \\
{[0.29 ; 0.72]}\end{array}$ & $\begin{array}{l}25 \\
(5.6)\end{array}$ & $51(2.5)$ & 0.002 \\
\hline Metabolic & $277(11.3)$ & $\begin{array}{l}<.0001 \\
\text { OR 0.32 } \\
{[0.25 ; 0.41]}\end{array}$ & $\begin{array}{l}90 \\
(20.2)\end{array}$ & $187(9.3)$ & $<.0001$ \\
\hline Neurological & $196(8.0)$ & $\begin{array}{l}<.0001 \\
\text { OR } 0.16 \\
{[0.11 ; 0.22]}\end{array}$ & $\begin{array}{l}51 \\
(11.5)\end{array}$ & $145(7.2)$ & 0.005 \\
\hline Pulmonary & 322 (13.1) & $\begin{array}{l}<.0001 \\
\text { OR 0.43 } \\
{[0.34 ; 0.55]}\end{array}$ & $\begin{array}{l}101 \\
(22.7)\end{array}$ & $\begin{array}{l}221 \\
(11.0)\end{array}$ & $<.0001$ \\
\hline Renal & $\begin{array}{l}1023 \\
(41.7)\end{array}$ & $\begin{array}{l}<.0001 \\
\text { OR } 0.60 \\
{[0.50 ; 0.70]}\end{array}$ & $\begin{array}{l}240 \\
(54.0)\end{array}$ & $\begin{array}{l}783 \\
(39.1)\end{array}$ & $<.0001$ \\
\hline \multicolumn{6}{|c|}{ * If statistically significant association with survival the odds ratio with confidence intervals are listed. } \\
\hline \multicolumn{6}{|c|}{$\begin{array}{l}\text { + Categorical variables expressed in numbers with percentages }[\mathrm{n}(\%)] \text { and continuous variables in } \\
\text { medians with interquartile ranges (median, [IQR]). }\end{array}$} \\
\hline $\begin{array}{l}\text { CNS: central ne } \\
\text { cardiopulmona } \\
\text { kg: kilograms, } \\
\text { positive inspira } \\
\text { venoarterial }\end{array}$ & $\begin{array}{l}\text { tem, ECMO: } \\
\text { tation, H1N1 } \\
\text { : sodium bic } \\
\text { ure, THAM: }\end{array}$ & $\begin{array}{l}\text { tracorporea } \\
\text { Jandemic in } \\
\text { bonate, paC } \\
\text { methamine }\end{array}$ & $\begin{array}{l}\text { mbrane } \\
\text { רza A H } \\
\text { oartial p } \\
\text { venoart }\end{array}$ & $\begin{array}{l}\text { ygenation, } \\
\text { subtype, IC } \\
\text { sure of arte } \\
\text { l, VV: veno }\end{array}$ & $\begin{array}{l}\text { CPR: ECMO } \\
\text { R: interquartile range, } \\
\text { al carbon dioxide, PIP: } \\
\text { enous, VVA: veno-- }\end{array}$ \\
\hline
\end{tabular}

There was no difference in the proportion of patients supported with veno-arterial (VA) vs veno-venous (VV) ECMO between groups, but more patients with $\mathrm{H} 1 \mathrm{~N} 1$ received ECMO for primary pulmonary indication than for seasonal influenza A $(97.5 \%$ vs $92.6 \%, p<0.0001)$, where the additional diagnosis of 
shock was also more common (17.2 vs $12.1 \%, \mathrm{p}=0.009)$ for influenza A vs H1N1 respectively. Patients with $\mathrm{H} 1 \mathrm{~N} 1$ were reported to experience more complications while on ECMO $(74.2 \%$ vs $56.4 \%, p<0.0001$, Table 1).

\section{Factors associated with survival to hospital discharge}

Univariate analyses are presented in Table 1. A priori selected variables from the univariate analysis were incorporated into a multivariate regression analysis with LASSO to associate with survival to hospital discharge in a predictive model (AUC 0.708, Table 2). Influenza A subtype was not associated with the primary outcome. Patient characteristics, including younger age (18-49 years vs others OR 2.57 (1.653.30)) and higher weight (OR $0.67(0.53-0.86))$ were associated with increased survival. Longer ventilation duration before ECMO (OR $0.53(0.40-0.72)$ ) and the use of metabolic buffer agents (OR 0.49 (0.35-0.67)) were associated with lower survival. Immunocompromised state (OR $0.54(0.37-0.81)$ ) and severity of illness at ECMO cannulation were also associated with lower survival, including pre-ECMO CNS dysfunction (OR $0.43(0.31-0.61)$ ), shock (OR $0.66(0.52-0.86))$ and cardiac arrest before ECMO (OR $0.39(0.27-0.56)$ ). 
Table 2

Predictive Model. Patient characteristics and pre-ECMO management associated with survival to hospital discharge by multivariable logistic regression with the least absolute shrinkage and selection operator (LASSO) predictive model.

\begin{tabular}{|c|c|c|}
\hline & OR [IQR] & $P$ value \\
\hline Influenza A vs. H1N1 & $0.83[0.63-1.23]$ & 0.1553 \\
\hline Male & $0.89[0.74-1.33]$ & 0.2896 \\
\hline Pre-ECMO arrest or ECPR & $0.39[0.27-0.56]$ & $<0.0001$ \\
\hline Neuromuscular blockade & $0.25[1.00-1.50]$ & 0.0258 \\
\hline Metabolic buffer agents & $0.49[0.35-0.67]$ & $<0.0001$ \\
\hline Non-respiratory co-infections & $0.91[0.70-3.09]$ & 0.6242 \\
\hline CNS dysfunction & $0.43[0.31-0.61]$ & $<0.0001$ \\
\hline Immunocompromised state & $0.54[0.37-0.81]$ & 0.0021 \\
\hline Shock & $0.66[0.52-0.86]$ & 0.0013 \\
\hline $\mathrm{PaCO}_{2} \geq 75 \mathrm{mmHg}$ & $0.84[0.66-1.23]$ & 0.1660 \\
\hline $\mathrm{PaCO}_{2}$ unknown & $0.91[0.71-2.88]$ & 0.5947 \\
\hline Age $18-49$ years & $2.57[1.65-3.30]$ & 0.0003 \\
\hline Age $50-59$ years & $1.18[0.60-1.54]$ & 0.3409 \\
\hline Intubation to time on ECMO $\geq 7$ days & $0.53[0.40-0.72]$ & $<0.0001$ \\
\hline Intubation to time on $\mathrm{ECMO} \geq 48$ hours $<7$ days & $0.66[0.53-0.83]$ & 0.0003 \\
\hline Intubation to time on ECMO unknown & $0.68[0.49-1.00]$ & 0.0241 \\
\hline Weight $\leq 75 \mathrm{~kg}$ & $0.67[0.53-0.86]$ & 0.0010 \\
\hline Weight 75-90 kg & $0.82[0.66-1.14]$ & 0.1060 \\
\hline \multicolumn{3}{|c|}{$\begin{array}{l}\text { CNS: central nervous system, ECMO: extracorporeal membrane oxygenation, ECPR: ECMO } \\
\text { cardiopulmonary resuscitation, H1N1: pandemic influenza A H1N1 subtype, IQR: interquartile range, } \\
\text { kg: kilograms, OR: odds ratio, paCO2: partial pressure of arterial carbon dioxide }\end{array}$} \\
\hline
\end{tabular}

For further assessment of explanatory factors during ECMO support associated with survival to hospital discharge, additional variables were incorporated into an explanatory multivariable model, including duration of ECMO run and complications sustained during ECMO (Table 3, AUC 0.785). There was no difference in survival according to Influenza A subtype across the study period. Patient characteristics, including younger age (18-49 years vs others OR 3.15 (2.17-4.15)) and higher weight (OR 0.67 (0.311.02)) were associated with higher survival. 
Table 3

Explanatory Model. Patient characteristics, pre-ECMO management, and ECMO run factors associated with survival to hospital discharge by multivariable logistic regression with the least absolute shrinkage and selection operator (LASSO) explanatory model with exact post-selection interference.

\begin{tabular}{|c|c|c|}
\hline & OR & $P$ value \\
\hline Influenza A vs. H1N1 & $1.25[0.38-1.73]$ & 0.4577 \\
\hline Male & $0.91[0.74-1.66]$ & 0.4456 \\
\hline Pre-ECMO arrest or ECPR & $0.52[0.35-0.86]$ & 0.0075 \\
\hline Neuromuscular blockade & $1.27[0.80-1.65]$ & 0.1369 \\
\hline Nitric oxide & $1.07[0.02-1.70]$ & 0.8235 \\
\hline Metabolic buffer agents & $0.60[0.41-0.91]$ & 0.0101 \\
\hline Cardiovascular complication & $0.70[0.39-0.88]$ & 0.0020 \\
\hline Hemorrhagic complication & $0.96[0.03-41.80]$ & 0.8889 \\
\hline Mechanical complication & $0.78[0.57-1.05]$ & 0.0452 \\
\hline Metabolic complication & $0.56[0.30-0.92]$ & 0.0137 \\
\hline Neurological complication & $0.17[0.11-0.24]$ & $<0.0001$ \\
\hline Pulmonary complication & $0.42[0.27-0.57]$ & $<0.0001$ \\
\hline Renal complication & $0.67[0.52-0.85$ & 0.0012 \\
\hline Non-respiratory co-infections & $0.93[0.81-5553.48]$ & 0.9301 \\
\hline CNS dysfunction & $0.80[0.54-1.93]$ & 0.3199 \\
\hline Immunocompromised & $0.53[0.35-0.83]$ & 0.0038 \\
\hline Shock & $0.78[0.56-1.10]$ & 0.0695 \\
\hline $\mathrm{PaCO}_{2} \geq 75 \mathrm{mmHg}$ & $0.89[0.68-1.84]$ & 0.4546 \\
\hline Age $18-49$ years & $3.15[2.17-4.15]$ & $<0.0001$ \\
\hline Age $50-59$ years & $1.39[0.94-1.87]$ & 0.0445 \\
\hline Intubation to time on $\mathrm{ECMO} \geq 7$ days & $0.58[0.31-0.81]$ & 0.0015 \\
\hline Intubation to time on ECMO $\geq 48$ hours $<7$ days & $0.72[0.53-0.94]$ & 0.0097 \\
\hline Intubation to time on ECMO unknown & $0.66[0.47-1.01]$ & 0.0265 \\
\hline
\end{tabular}

CNS: central nervous system, ECMO: extracorporeal membrane oxygenation, ECPR: ECMO cardiopulmonary resuscitation, H1N1: pandemic influenza A H1N1 subtype, IQR: interquartile range, kg: kilograms, OR: odds ratio, paCO2: partial pressure of arterial carbon dioxide 


\begin{tabular}{|lcc|}
\hline & OR & P value \\
\hline Weight $\leq 75 \mathrm{~kg}$ & $0.67[0.31-1.02]$ & 0.0296 \\
\hline Weight $\mathbf{7 5}-\mathbf{9 0} \mathrm{kg}$ & $0.87[0.47-1.81]$ & 0.3524 \\
\hline Weight $\mathbf{9 0 - 1 1 0 ~ k g}$ & $1.09[0.33-1.60]$ & 0.6092 \\
\hline Hours ECMO $\mathbf{4 4 2 . 5}$ & $2.50[1.26-3.67]$ & 0.0070 \\
\hline Hours ECMO 256.0-442.5 & $2.73[1.65-3.76]$ & 0.0005 \\
\hline Hours ECMO 146.5-256.0 & $3.09[2.25-4.10]$ & $<0.0001$ \\
\hline $\begin{array}{l}\text { CNS: central nervous system, ECMO: extracorporeal membrane oxygenation, ECPR: ECMO } \\
\text { cardiopulmonary resuscitation, H1N1: pandemic influenza A H1N1 subtype, IQR: interquartile range, } \\
\text { kg: kilograms, OR: odds ratio, paCO2: partial pressure of arterial carbon dioxide }\end{array}$ & \\
\hline
\end{tabular}

Patient management, including longer ventilation duration before ECMO (OR 0.58 (0.31-0.0.81)) and the use of metabolic buffer agents (OR $0.60(0.41-0.91))$ were associated with lower survival. Immunocompromised state (OR $0.53(0.35-0.85)$ ) and severity of illness at ECMO cannulation were also associated with lower survival, including cardiac arrest before ECMO (OR 0.52 (0.35-0.86)). Patients with the shortest ECMO runs ( $<146.5$ hours) were more likely to be associated with lower survival than those with longer ECMO runs (OR 3.09 (2.25-4.10)). ECMO complications except bleeding were associated with reduced survival $(p<0.001)$.

\section{Discussion}

This study demonstrates that patients supported on ECMO for pandemic $\mathrm{H} 1 \mathrm{~N} 1$ had more severe features of critical illness, despite being younger, with higher weight and having fewer comorbidities than those subsequently managed on ECMO for seasonal influenza A. These findings support increased virulence associated with novel virus triggering the pandemic, but may additionally reflect resource limitation of this invasive support during the associated abrupt increase in critical care utilization. Importantly, despite differences in severity of illness, there was no difference in survival to hospital discharge for those patients with pandemic $\mathrm{H} 1 \mathrm{~N} 1$ compared with patients subsequently managed on ECMO with seasonal influenza A. We did identify patient characteristics, aspects of patient management before ECMO, and ECMO complications that were associated with survival to hospital discharge.

Igniting the surge in ECMO use for adults with ARDS was the success of ECMO during the H1N1 pandemic $(2,3,17-25)$. Our study demonstrates continued ECMO use after the 2009 pandemic, more for seasonal influenza A subtypes than H1N1. Despite the higher severity of illness in the H1N1 patients, we did not find a difference in survival according to viral subtype. Studies evaluating the use of ECMO for other viral etiologies of ARDS continue to emerge (33-38). Since the novel COVID-19 pandemic, investigators have reported successful ECMO support with similar survival to hospital discharge, even when directly compared with influenza cohorts $(36,37)$. 
ECMO support for ARDS continued to evolve after the H1N1 pandemic. A single-center study reported up to $80 \%$ survival for $\mathrm{H} 1 \mathrm{~N} 1$ patients supported on ECMO during 2013-2014 (39). Studies from Japan and Korea demonstrated improved outcomes during a resurgence of H1N1 in 2016 when compared to the 2009 pandemic, which likely reflects improvements in their patient selection and management $(40,41)$. Our study found that, overall, there was no difference in survival in the pandemic $\mathrm{H} 1 \mathrm{~N} 1$ subtype patients supported on ECMO during the 2009 pandemic year compared to years thereafter. The abrupt increase in hospitalizations and ECMO use during the 2009 pandemic reflected intensified virulence and amplification of the novel H1N1 virus in the community, which highlights the capacity to surge and allocate resources appropriately to support ECMO patients when needed (10).

Allocation of scarce resources or complex resource-intensive therapies during a pandemic can, however, become problematic. Identification of patient factors, as well as patient management strategies prior to ECMO which may be associated with improved outcomes, can inform prioritization during times of limited resource availability. Many of the mortality prediction scores created to help determine ECMO candidacy were developed using patients during the H1N1 pandemic, and thus, it is not surprising that we have identified similar clinical characteristics as associated with survival to hospital discharge $(24,29$, $42,43)$. However, the majority of our patients had seasonal influenza and not specifically H1N1, and thus factors associated with mortality in our predictive and explanatory models may be more applicable to other viral subtypes causing ARDS. As in previous studies, younger age, higher weight, and lack of reported comorbidities were associated with survival $(2,6,19,22,32,34,42)$. Additionally, those patients who were managed with a shorter duration of mechanical ventilation, who had not progressed to cardiac arrest prior to ECMO cannulation were found to have improved survival, supporting early initiation of ECMO for viral $\operatorname{ARDS}(3,6,18,29,42)$. Established ECMO programs with integrated systems to prevent and mitigate complications may be best placed to offer this invasive support, even during times of pandemic-associated resource limitation $(37,44)$.

\section{Study limitations}

Our study has the expected limitations inherent in a retrospective observational study. ELSO Registry data is entered voluntarily, without external validation of data in the represented era, however, the institution of a data dictionary, data entry exam, and logic-limited data entry has resulted in improved data quality in the ELSO registry over the duration of this study (45). Our data may be subject to era effect. Some unidentified confounding covariates, such as the older population's prior exposure to H1N1, may impact our results. Our application of LASSO regression adjusting for predefined comorbidities used in the RESP score is a strength of our analysis; however, we did not specifically include other potential comorbidities $(29,46)$. Additionally, clinically relevant covariates that had more than $15 \%$ missing data were excluded from the analysis.

\section{Conclusions}


Over the last decade, the utilization of ECMO for viral ARDS has become well established. In this study of patients with Influenza A supported with ECMO, those with pandemic $\mathrm{H} 1 \mathrm{~N} 1$ were younger, with more severe illness than those supported for seasonal influenza A. Survival to hospital discharge was associated with patient characteristics, management, and ECMO complications, but was not impacted by the specific influenza A subtype. Identification of these factors may inform patient selection and preECMO management, which is especially important in the setting of resource limitation.

\section{Declarations}

Ethics approval and consent to participate: Permission to analyze the data was granted by the Extracorporeal Life Support Organization Scholarly Oversite Committee and consent was exempted by the Institutional Review Board of Baylor College of Medicine, Houston, Texas, USA.

Consent for publication: Exempted by the Institutional Review Board of Baylor College of Medicine, Houston, Texas, USA.

Availability of data and materials: The data that support the findings of this study are available from the Extracorporeal Life Support Organization Scholarly Oversite Committee with submission of the written request form.

Competing interests: PA reports grants from the National Institutes of Health $(\mathrm{NIH})$ to support research activities not specific to this study (1R13HD104432-01 Pediatric ECMO Anticoagulation CollaborativE PEACE). JET is supported by a Career Development Award from the National Institutes of Health/National Heart, Lung, And Blood Institute (K23 HL141596). JET received speaker fees and travel compensation from LivaNova and Philips Healthcare, unrelated to this work. RPB reports grants from National Institutes of Health (R01 HL153519-ASCEND; K12 HL138039-TACTICAL; R01 HD01543-Pediatric Implantable Artificial Lung) outside the submitted work; RPB also discloses that he is the Extracorporeal Life Support Organization (ELSO) Registry Chair. All other authors have no conflicts of interest to disclose (EO, MA, ML, HL,JL, PR, GL, and LS).

Funding: Internal funding was secured for this project.

Authors' contributions: Study conception, design, material preparation, data collection and analysis were performed by MA, EO, and PA. ML conceived and supervised the statistical analysis. $\mathrm{HL}, \mathrm{JA}$, and ML performed the statistical analysis. The first draft of the manuscript was written by EO, and all authors revised the manuscript for important intellectual content. All authors read and approved the final manuscript.

Acknowledgements: None.

\section{References}


1. Brodie D, Slutsky AS, Combes A. Extracorporeal life support for adults with respiratory failure and related indications: a review. JAMA. 2019;322:557-568.

2. Zangrillo A, Biondi-Zoccai G, Landoni G, Frati G, Patroniti N, Pesenti A, Pappalardo F. Extracorporeal membrane oxygenation (ECMO) in patients with $\mathrm{H} 1 \mathrm{~N} 1$ influenza infection: a systematic review and meta-analysis including 8 studies and 266 patients receiving ECMO. Crit Care. 2013;17(1):R30.

3. Sukhal S, Sethi J, Ganesh M, Villablanca PA, Malhotra AK, Ramakrishna H. Extracorporeal membrane oxygenation in severe influenza infection with respiratory failure: a systematic review and metaanalysis. Ann Card Anaesth. 2017;20:14-21.

4. Zapol WM, Snider MT, Hill JD, Fallat RJ, Bartlett RH, Edmunds LH, et al. Extracorporeal membrane oxygenation in severe acute respiratory failure. A randomized prospective study. JAMA. 1979;42:2193-2196.

5. Morris AH, Wallace CJ, Menlove RL, Clemmer TP, Orme JF, Weaver LK, et al. Randomized clinical trial of pressure-controlled inverse ratio ventilation and extracorporeal $\mathrm{CO} 2$ removal for adult respiratory distress syndrome. Am J Respir Crit Care Med. 1994;149:295-305.

6. Brogan TV, Thiagarajan RR, Rycus PT, Bartlett RH, Bratton SL. Extracorporeal membrane oxygenation in adults with severe respiratory failure: a multi-center database. Intensive Care Med. 2009;35:21052114.

7. Peek GJ, Mugford M, Tiruvoipati R, Wilson A, Allen E, Thalanany MM, et al. Efficacy and economic assessment of conventional ventilatory support versus extracorporeal membrane oxygenation for severe adult respiratory failure (CESAR): a multicentre randomised controlled trial. Lancet. 2009;374:1351-1363.

8. Combes A, Hajage D, Capellier G, Demoule A, Lavoue S, Guervilly C, et al. Extracorporeal membrane oxygenation for severe acute respiratory distress syndrome. N Engl J Med. 2018; 378:1965-1975.

9. Goligher EC, Tomlinson G, Hajage D, Wijeysundera DN, Fan E, Jüni P, et al. Extracorporeal membrane oxygenation for severe acute respiratory distress syndrome and posterior probability of mortality benefit in a post hoc Bayesian analysis of a randomized clinical trial. JAMA. 2018;320:2251-2259.

10. Chang Y, van Hal SJ, Spencer PM, Gosbell IB, Collett PW. Comparison of adult patients hospitalised with pandemic (H1N1) 2009 influenza and seasonal influenza during the "PROTECT" phase of the pandemic response. Med J Aust. 2010;192:90-93.

11. von der Beck D, Seeger W, Herold S, Günther A, Löh B. Characteristics and outcomes of a cohort hospitalized for pandemic and seasonal influenza in Germany based on nationwide inpatient data. PLoS One. 2017;12:e0180920.

12. Belongia EA, Irving SA, Waring SC, Coleman LA, Meece JK, Vandermause M, et al. Clinical characteristics and 30-day outcomes for influenza A 2009 (H1 N1), 2008-2009 (H1N1), and 20072008 (H3N2) infections. JAMA. 2010;304:1091-1098.

13. Reed C, Chaves SS, Perez A, D'Mello T, Daily Kirley P, Aragon D, et al. Complications among adults hospitalized with influenza: a comparison of seasonal influenza and the $2009 \mathrm{H} 1 \mathrm{~N} 1$ pandemic. Clin Infect Dis. 2014;59:166-174. 
14. Carcione D, Giele C, Dowse GK, Mak DB, Goggin L, Kwan K, et al. Comparison of pandemic (H1N1) 2009 and seasonal influenza, Western Australia, 2009. Emerg Infect Dis. 2010;16:1388-1395.

15. Lemaitre $M$, Carrat F. Comparative age distribution of influenza morbidity and mortality during seasonal influenza epidemics and the 2009 H1N1 pandemic. BMC Infect Dis. 2010;10:162-166.

16. Khandaker G, Dierig A, Rashid H, King C, Heron L, Booy R. Systematic review of clinical and epidemiological features of the pandemic influenza A (H1N1) 2009. Influenza Other Respir Viruses. 2011;5:148-156.

17. Holzgraefe B, Broome M, Kalzen H, Konrad D, Palmer K, Frenckner B. Extracorporeal membrane oxygenation for pandemic H1N1 2009 respiratory failure. Minerva Anestesiol. 2010;76:1043-1051.

18. Patroniti N, Zangrillo A, Pappalardo F, Peris A, Cianchi G, Braschi A, et al. The Italian ECMO network experience during the 2009 infuenza $A(H 1 N 1)$ pandemic: preparation for severe respiratory emergency outbreaks. Intensive Care Med. 2011;37:1447-1457.

19. Australia and New Zealand Extracorporeal Membrane Oxygenation (ANZ ECMO) Influenza Investigators, Davies A, Jones D, Bailey M, Beca J, Bellomo R, et al. Extracorporeal membrane oxygenation for 2009 influenza $A(\mathrm{H} 1 \mathrm{~N} 1)$ acute respiratory distress syndrome. JAMA. 2009;302:1888-1895.

20. Noah MA, Peek GJ, Finney SJ, Grifths MJ, Harrison DA, Grieve R, et al. Referral to an extracorporeal membrane oxygenation center and mortality among patients with severe 2009 influenza A(H1N1). JAMA. 2011;306:1659-1668.

21. Takeda S, Kotani T, Nakagawa S, Ichiba S, Aokage T, Ochiai R, et al. Extracorporeal membrane oxygenation for 2009 influenza A(H1N1) severe respiratory failure in Japan. J Anesth. 2012;26:6507.

22. Pham T, Combes A, Rozé $H$, Chevret $S$, Mercat $A$, Roch $A$, et al. Extracorporeal membrane oxygenation for pandemic influenza $A(\mathrm{H} 1 \mathrm{~N} 1)$-induced acute respiratory distress syndrome: a cohort study and propensity-matched analysis. Am J Respir Crit Care Med. 2013;187:276 - 85.

23. Weber-Carstens S, Goldmann A, Quintel M, Kalenka A, Kluge S, Peters J, et al. Extracorporeal lung support in H1N1 provoked acute respiratory failure: the experience of the German ARDS Network. Dtsch Arztebl Int. 2013;110:543-9.

24. Roch A, Lepaul-Ercole R, Grisoli D, Bessereau J, Brissy O, Castanier M, et al. Extracorporeal membrane oxygenation for severe influenza A (H1N1) acute respiratory distress syndrome: a prospective observational comparative study. Intensive Care Med. 2010;36:1899 - 905.

25. Bonastre J, Suberviola B, Pozo JC, Guerrero JE, Torres A, Rodríguez A, et al. [Extracorporeal lung support in patients with severe respiratory failure secondary to the 2010-2011 winter seasonal outbreak of influenza A (H1N1) in Spain]. Med Intensiva. 2012;36:193-9.

26. Hou X, Guo L, Zhan Q, Jia X, Mi Y, Li B, et al. Extracorporeal membrane oxygenation for critically ill patients with 2009 influenza $A(\mathrm{H} 1 \mathrm{~N} 1)$-related acute respiratory distress syndrome: preliminary experience from a single center. Artif Organs. 2012;36:780-6. 
27. Beurtheret S, Mastroianni C, Pozzi M, D'Alessandro C, Luyt CE, Combes A, et al. Extracorporeal membrane oxygenation for 2009 influenza A (H1N1) acute respiratory distress syndrome: singlecentre experience with 1-year follow-up. Eur J Cardiothorac Surg. 2012;41:691-5.

28. Hodgson CL, Hayes K, Everard T, Nichol A, Davies AR, Bailey MJ, et al. Long-term quality of life in patients with acute respiratory distress syndrome requiring extracorporeal membrane oxygenation for refractory hypoxaemia. Crit Care. 2012;16:R202.

29. Schmidt M, Bailey M, Sheldrake J, Hodgson C, Aubron C, Rycus PT, et al. Predicting survival after extracorporeal membrane oxygenation for severe acute respiratory failure. The Respiratory Extracorporeal Membrane Oxygenation Survival Prediction (RESP) score. Am J Respir Crit Care Med. 2014;189:1374-82.

30. Extracorporeal Life Support Organization. ECLS Registry Report. International Summary, July 2020. https://www.elso.org/registry/statistics/InternationalSummary.aspx. Accessed 11 Jan 2021.

31. Leisman DE, Harhay MO, Lederer DJ, Abramson M, Adjei AA, Bakker J, et al. Development and reporting of prediction models: Guidance for authors from editors of respiratory, sleep, and critical care journals. Crit Care Med. 2020;48:623-633.

32. Lee JD, Sun DL, Sun Y, Taylor JE. Exact post-selection inference, with application to the lasso. Ann Statist. 2016;44:907-927.

33. Buchner J, Mazzeffi M, Kon Z, Menaker J, Rubinson L, Bittle G, et al. Single-center experience with venovenous ECMO for influenza-related ARDS. J Cardiothorac Vasc Anesth. 2018;32:1154-1159.

34. Kraef C, van der Meirschen M, Wichmann D, Kutza M, Restemeyer C, Addo MM, et al. [Management of seasonal influenza in 2017/2018 at a German tertiary-care hospital]. Bundesgesundheitsblatt Gesundheitsforschung Gesundheitsschutz. 2019;62:870-880.

35. Huang L, Zhang W, Yang Y, Wu W, Lu W, Xue H, et al. Application of extracorporeal membrane oxygenation in patients with severe acute respiratory distress syndrome induced by avian influenza $A$ (H7N9) viral pneumonia: national data from the Chinese multicentre collaboration. BMC Infect Dis. 2018;18:23.

36. Cousin N, Bourel C, Carpentier D, Goutay J, Mugnier A, Labreuche J, et al. SARS-CoV-2 versus influenza-associated acute respiratory distress syndrome requiring veno-venous extracorporeal membrane oxygenation support. ASAIO J. 2021;67:125-131.

37. Barbaro RP, MacLaren G, Boonstra PS, Iwashyna TJ, Slutsky AS, Fan E, et al. Extracorporeal membrane oxygenation support in COVID-19: an international cohort study of the Extracorporeal Life Support Organization registry. Lancet. 2020;396:1071-1078.

38. Vandroux D, Kerambrun H, Ferdynus C, Allou N, Allyn J, Gaüzère BA, et al. Postpandemic influenza mortality of venovenous extracorporeal membrane oxygenation-treated patients in Reunion Island: a retrospective single center study. J Cardiothorac Vasc Anesth. 2020;34:1426-1430.

39. Menon N, Perez-Velez CM, Wheeler JA, Morris MF, Amabile OL, Tasset MR, et al. Extracorporeal membrane oxygenation in acute respiratory distress syndrome due to influenza A (H1N1)pdm09 
pneumonia. A single-center experience during the 2013-2014 season. Rev Bras Ter Intensiva. 2017;29:271-278.

40. Ohshimo S, Shime N, Nakagawa S, Nishida O, Takeda S; Committee of the Japan ECMO project. Comparison of extracorporeal membrane oxygenation outcome for influenza-associated acute respiratory failure in Japan between 2009 and 2016. J Intensive Care. 2018;6:38.

41. Choi H, Ko UW, Lee H, Hong SB, Chung CR. Improved survival rates in patients with H1N1 acute respiratory failure in Korea between 2009 and 2016. PLoS One. 2019;14:e0223323.

42. Schmidt $M$, Zogheib E, Rozé $H$, Repesse X, Lebreton G, Luyt CE, et al. The PRESERVE mortality risk score and analysis of long-term outcomes after extracorporeal membrane oxygenation for severe acute respiratory distress syndrome. Intensive Care Med. 2013;39:1704-13.

43. Pappalardo F, Pieri M, Greco T, Patroniti N, Pesenti A, Arcadipane A, et al. Predicting mortality risk in patients undergoing venovenous ECMO for ARDS due to influenza A (H1N1) pneumonia: the ECMOnet score. Intensive Care Med. 2013;39:275 - 81.

44. Barbaro RP, Odetola FO, Kidwell KM, Paden ML, Bartlett RH, Davis MM, et al. Association of hospitallevel volume of extracorporeal membrane oxygenation cases and mortality. Analysis of the extracorporeal life support organization registry. Am J Respir Crit Care Med. 2015;191:894-901.

45. Lorusso R, Alexander P, Rycus P, Barbaro R. The Extracorporeal Life Support Organization Registry: update and perspectives. Ann Cardiothorac Surg. 2019;8:93-98.

46. Elixhauser A, Steiner C, Harris DR, Coffey RM. Comorbidity measures for use with administrative data. Med Care. 1998;36:8-27.

\section{Figures}


Extracorporeal Life Society Organization Registry

January 01, 2009 - December 31, 2019

2,515 patients $>=18$ years with Diagnosis of Influenza A or H1N1

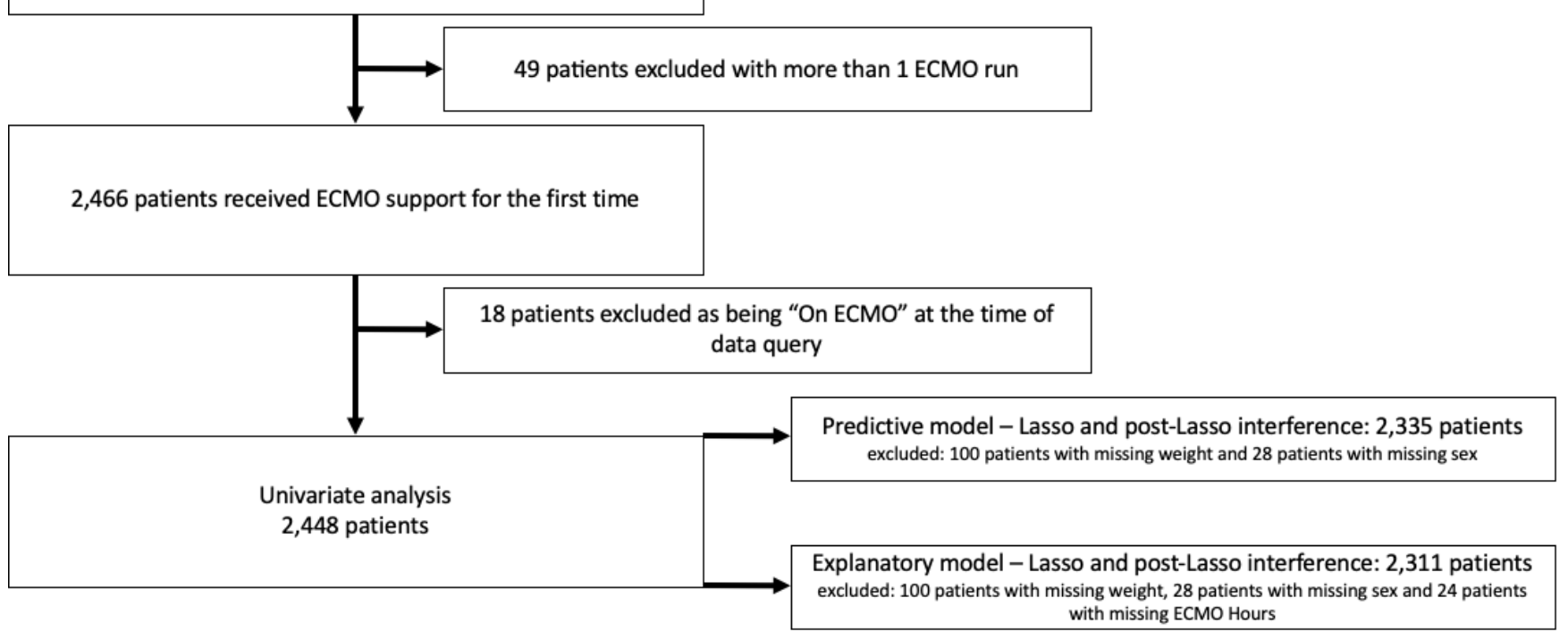

\section{Figure 1}

Study Flowchart ECMO: extracorporeal membrane oxygenation, H1N1: pandemic influenza A H1N1 subtype, LASSO: least absolute shrinkage and selection operator 


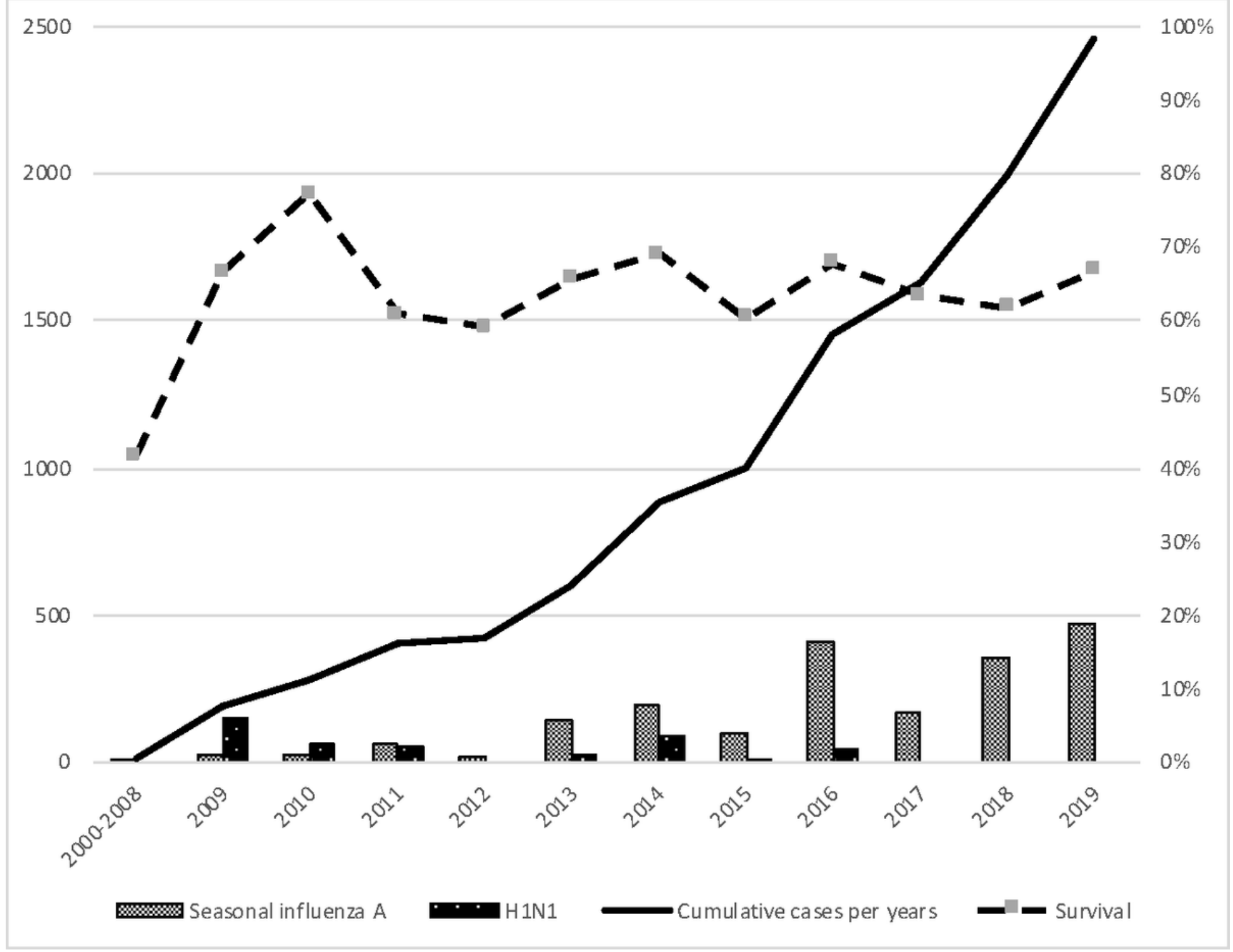

Figure 2

Survival and number of seasonal influenza A patients and H1N1 patients over the study period. H1N1: pandemic influenza A H1N1 subtype

\section{Supplementary Files}

This is a list of supplementary files associated with this preprint. Click to download.

- InfluenzaAECMOOnlineSupplements.docx 